Relations industrielles

Industrial Relations

\title{
L'état de la discipline en relations industrielles au Canada: Un brin d'histoire et une postface
}

\section{Gérard Hébert}

Volume 44, numéro 4, 1989

URI : https://id.erudit.org/iderudit/050539ar

DOI : https://doi.org/10.7202/050539ar

Aller au sommaire du numéro

Éditeur(s)

Département des relations industrielles de l'Université Laval

ISSN

0034-379X (imprimé)

1703-8138 (numérique)

Découvrir la revue

Citer cet article

Hébert, G. (1989). L'état de la discipline en relations industrielles au Canada: Un brin d'histoire et une postface. Relations industrielles / Industrial Relations, 44(4), 884-904. https://doi.org/10.7202/050539ar
Résumé de l'article

L'état de la discipline en relations industrielles au Canada : un brin d'histoire et une postface.
Tous droits réservés (c) Département des relations industrielles de l'Université Laval, 1989
Ce document est protégé par la loi sur le droit d'auteur. L'utilisation des services d'Érudit (y compris la reproduction) est assujettie à sa politique d'utilisation que vous pouvez consulter en ligne.

https://apropos.erudit.org/fr/usagers/politique-dutilisation/ 


\title{
DISCUSSION
}

\section{L'état de la discipline en relations industrielles au Canada}

\author{
Un brin d'histoire et une postface
}

\section{Gérard Hébert}

L'Association canadienne de relations industrielles (ACRI) a publié, au cours de la dernière année académique, un ouvrage composé d'une série d'études sur L'état de la discipline en relations industrielles au Canada. Le livre a paru dans les deux langues officielles du pays, soit le français et l'anglais ${ }^{1}$.

Le présent exposé comportera d'abord quelques notes historiques sur les péripéties qui ont marqué la préparation de l'ouvrage, quelques commentaires sur les principaux problèmes soulevés par sa réalisation, et, enfin, les prochaines étapes à envisager dans la poursuite d'une théorie des relations industrielles.

\section{HISTOIRE EXTERNE DU LIVRE}

Le volume est demeuré en chantier pendant près de 10 ans. L'idée première a surgi d'un rapport que le professeur Thomas Kochan, du Massachussetts Institute of Technology, préparait alors pour le gouvernement américain ${ }^{2}$. Le professeur Noah Meltz de l'Université de Toronto a approché des représentants du ministère fédéral du Travail pour discuter de la possibilité d'entreprendre une étude semblable pour le Canada. À son assemblée annuelle, en 1980, l'ACRI donnait à son comité exécutif le mandat de poursuivre le projet. Quelques mois plus tard, le Comité sur les politiques

* HÉBERT, G., professeur, École de relations industrielles, Université de Montréal.

1 L'état de la discipline en relations industrielles au Canada, sous la direction de Gérard HÉBERT, Hem C. JAIN et Noah M. MELTZ. Études réalisées sous les auspices de l'Association canadienne de relations industrielles, Université de Montréal, École de relations industrielles, 1988, xxii - $372 \mathrm{p}$. The State of the Art in Industrial Relations. A project of the Canadian Industrial Relations Association. Edited by Gérard HÉBERT, Hem C. JAIN and Noah M. MELTZ. Published by the Industrial Relations Centre of Queen's University at Kingston and the Centre for Industrial Relations at the University of Toronto, C. 1988, xx - $301 \mathrm{p}$.

2 Thomas A. KOCHAN, Labor-Management Relations Research Priorities for the 1980s, Washington D.C., U.S. Department of Labor, January 1980. 
de recherches présentait un projet grandiose, qui impliquait des dépenses de près de $\$ 200000$. Quelques mois plus tard, Travail Canada donnait sa réponse: elle était négative.

Le Comité a décidé alors de soumettre le projet, avec une demande de subvention, au Conseil de recherches en sciences humaines du Canada. Le Conseil avait manifesté de l'intérêt pour des projets sur l'état des différentes disciplines en sciences humaines. Un an plus tard, le Conseil offrait la maigre subvention de $\$ 15000$, laquelle ne fut effectivement versée qu'une année plus tard, au début de 1983. L'argent a servi à payer des assistants de recherches, à financer le travail de dactylo, les photocopies, les appels téléphoniques interurbains et quelques rares frais de déplacement. Le projet a donc été réalisé par la contribution volontaire de tous les collaborateurs, à commencer par les membres du Comité de direction.

Des retards incontrôlables sont survenus. La mise au point du plan du livre et le recrutement des personnes compétentes pour en écrire les différents chapitres ont pris plus de temps que prévu. Le projet s'est vraiment mis en route en juin 1984, à l'occasion de l'assemblée annuelle de l'ACRI à Guelph en Ontario. Nous pensions tous alors qu'une année ou deux suffiraient pour la composition et la publication du livre. Comme il arrive bien souvent dans de tels projets, un ou deux chapitres ont retardé le tout, et la date de publication s'en est trouvée ainsi plusieurs fois reportée. Le volume a été finalement lancé en août 1988, à l'occasion du $1^{\text {er }}$ Congrès de relations industrielles des Amériques, à Québec. La version française du volume était alors prête et disponible; le volume anglais parut quelques mois plus tard.

Voilà un résumé bien bref d'une longue histoire, difficile à certains moments. Le côté intéressant et enrichissant du projet se manifestait, entre autres, dans les nombreuses discussions que le Comité de direction dut avoir pour faire les choix requis dans cette longue entreprise.

\section{HISTOIRE INTERNE DU LIVRE}

Les choix à faire portaient sur bien des aspects. Je m'arrêterai à trois d'entre eux d'importance primordiale: la définition des relations industrielles, la manière d'aborder l'état de la discipline et finalement le plan du volume.

\section{Définition}

Le premier problème que nous avons dû envisager était aussi fondamental que de savoir comment nous allions définir les relations industrielles. Même en partant de données empiriques, comme les structures universitaires et la manière d'enseigner cette matière, nous étions en présence d'une dualité fondamentale. Les universités de langue anglaise au Canada ont tendance à avoir des centres de relations industrielles, dont la préoccupation principale vise les relations collectives de travail, soit le syndicalisme, le droit du travail et la négociation collective; l'enseignement de la direction du personnel ou de la gestion des ressources humaines est généralement 
laissé aux écoles ou aux facultés d'administration ou de management. D'un autre côté, les universités francophones ont choisi la formule opposée: elles ont mis sur pied des départements de relations industrielles, où l'on enseigne à la fois les rapports collectifs et la direction du personnel ou la gestion des ressources humaines; comme départements, ces unités ont leur corps professoral et leur propre groupe d'étudiants officiellement inscrits comme étudiants de relations industrielles.

Le Comité de direction s'est vite mis d'accord que les deux aspects devaient faire partie de la définition adoptée dans le volume. Mais, pour que le livre s'inspire vraiment d'une telle définition, il ne suffisait pas d'une déclaration à cet effet dans le chapitre d'ouverture. Malgré cette prise de position, chaque auteur demeurait libre d'aborder son chapitre selon sa propre perception, dans l'optique qui lui était familière par suite de son enseignement et de ses travaux de recherche. C'est ainsi que le livre, surtout dans les milieux francophones, a été accusé d'avoir accordé trop d'importance aux rapports collectifs et vraiment très peu, selon certains, à la place et au rôle de la gestion des ressources humaines.

\section{Approche}

Le second problème du Comité de direction était peut-être de moindre envergure, mais il n'était pas plus facile à résoudre. Tout le monde reconnaît qu'il s'agit là d'un champ d'études privilégié pour une approche multidisciplinaire, mais l'unanimité ne va guère plus loin. Pas difficile de s'entendre sur le fait que quelques disciplines d'importance majeure devaient faire partie du projet, mais la manière de les intégrer paraissait beaucoup moins claire. Très tôt, nous fûmes confrontés au choix suivant: nous pouvions choisir un certain nombre de questions ou problèmes et demander à chaque auteur d'en discuter selon une approche multidisciplinaire; ou nous pouvions choisir l'approche par discipline et suggérer à chaque auteur d'inclure dans sa présentation de la discipline et de ses rapports avec les relations industrielles les sujets principaux qu'elle avait étudiés ou qu'elle aurait dû considérer.

Le Comité de direction conclut que la seconde méthode comportait moins de risque: il lui parut presque impossible d'établir une liste de sujets ou de problèmes qui auraient une chance de faire une certaine unanimité parmi les spécialistes de relations industrielles. Il lui restait une troisième tâche, qui comportait elle aussi des choix difficiles, celle de déterminer les disciplines qu'on allait inclure et d'établir par le fait même le plan de l'ouvrage.

\section{Le plan}

Il allait de soi que certaines disciplines devaient occuper une place de choix dans l'ouvrage. L'économique et le droit ont toujours été les deux disciplines les plus étroitement liées aux relations industrielles tout au long du $\mathrm{XX}^{\mathrm{e}}$ siècle. Plus récemment, la sociologie et la psychologie, et leur fils cadet 
le behaviorisme, ont contribué de façon significative à la compréhension des relations industrielles et, à ce titre, devaient faire partie du noyau central des disciplines à aborder.

Mais l'unanimité s'arrêtait là. Qu'en serait-il, par exemple, des sciences de l'administration, de l'histoire et de la philosophie pour ne nommer que ces trois-là? Tous ne considéraient pas les études en administration comme une discipline particulière et reconnue; certains soutenaient que ces études occupent exactement la même position par rapport aux autres que les relations industrielles. Le Comité finit par conclure qu'il fallait tout de même inclure un chapitre sur les études d'administration et sur l'histoire, à cause de la contribution remarquable de ces deux secteurs à l'intelligence de certains aspects des relations du travail. Mais le Comité n'a pas retenu la philosophie, non pas que le sujet ne soit pas important: les options fondamentales et les valeurs se retrouvent dans tous les problèmes de relations industrielles. Le Comité a plutôt fondé sa décision sur la difficulté, voire l'impossibilité, de trouver quelqu'un qui puisse présenter une vue d'ensemble du sujet - la philosophie, incluant l'éthique des comportements, et les relations industrielles - sans indisposer la moitié sinon la totalité de ses lecteurs. Le Comité a estimé que le sujet était trop délicat et qu'il fallait l'éviter dans une première tentative de cette nature. Car c'était bien un projet d'association que nous avions accepté de réaliser.

À l'inverse, le Comité a inclus un chapitre sur les aspects principaux des relations industrielles - en anglais mainstream industrial relations - parce que plusieurs sont d'avis que certains sujets ne peuvent être traités de façon adéquate par aucune des principales disciplines de sciences sociales. Un peu dans le même sens, le Comité considérait qu'il ne pouvait ignorer l'approche systémique, si importante en relations industrielles, dans un ouvrage sur l'état de la discipline. Le Comité a donc invité, pour écrire ce chapitre, un auteur souvent cité pour avoir poussé plus loin le modèle établi il y a plus de 30 ans par John T. Dunlop ${ }^{3}$.

Un dernier mot sur le plan du livre. Le Comité a estimé que deux des disciplines retenues devaient comporter chacune deux chapitres, un sur le Canada français et un sur le Canada anglais, à cause de leur relation étroite et essentielle avec les aspects des cultures différentes impliquées, soit le droit et l'histoire. En d'autres mots, si on ajoute les chapitres d'introduction et de conclusion, l'ouvrage en contient dix pour traiter de cinq disciplines et de leurs rapports aux relations industrielles.

\section{Une discipline ou un champ d'études?}

La question est demeurée sans réponse, et le Comité a conclu qu'il devait en être ainsi. Dans les premières étapes du projet, en 1983, je me suis porté volontaire pour écrire une introduction qui suggérerait les principales orientations de l'ouvrage; elle fut soumise à l'assemblée annuelle de l'ACRI à Vancouver. Je ne pouvais être présent à cette assemblée. On m'a dit que la

3 John T. DUNLOP, Industrial Relations Systems, New York, Henry Holt, 1958, $339 \mathrm{p}$. 
discussion entre les partisans de la discipline et les partisans du champ d'études devint si intense, pour ne pas dire si violente, que le projet luimême s'en est presque trouvé menacé.

Il n'était pas possible de ne pas faire mention du problème, à cause de son caractère très fondamental. Nous étions d'accord que le problème devait être présenté dans le chapitre d'introduction. Chaque auteur était libre de discuter de la question s'il désirait le faire. La rédaction du chapitre de conclusion demeurait beaucoup plus difficile. Malgré mon opinion personnelle sur le sujet, j'ai tâché de présenter les deux points de vue avec toute l'honnêteté et la force possible pour chacun des points de vue. Un projet d'association ne pouvait courir le risque de se mettre à dos une partie importante de ses membres en prenant une position tranchée sur une question aussi controversée.

\section{ÉTAT DE LA DISCIPLINE}

En quoi devait consister principalement une étude sur l'état de la discipline? La réponse n'était pas sans soulever certaines questions. Quand le projet fut mis en route, d'autres secteurs des sciences sociales s'étaient déjà engagés dans cette voie ${ }^{4}$, mais très peu de publications sur le sujet avaient encore vu le jour ${ }^{5}$. Après de longues discussions, nous finîmes par conclure qu'un état de la discipline relativement complet devait inclure l'état des connaissances dans le secteur, un état de la théorie et un état de la recherche. Chaque auteur était libre d'insister sur tel ou tel aspect, à son choix, mais le Comité avait défini l'état de la discipline comme incluant idéalement les trois aspects.

\section{État des connaissances et de la recherche}

On trouve dans le volume une bonne description de l'état des connaissances et de l'état de la recherche en relations industrielles, selon chacune des disciplines envisagées. Ce sont, me semble-t-il, deux des points forts du volume.

4 Deux de ces études devaient paraître par la suite sous les titres suivants. Le droit et le savoir, Rapport au Conseil de recherches en sciences humaines du Canada par le Groupe consultatif sur la recherche et les études en droit, Ottawa, Conseil de recherches en sciences humaines du Canada, 1988, x-212 p. The Development of Political Science in Canada: Fifty Years in the History of a Discipline, publié sous la direction de Michael STEIN, John TRENT et André DONNEUR.

5 Groupe consultatif sur la recherche et l'éducation supérieure dans les études de la gestion et de l'administration, Les études et les recherches universitaires en gestion: une situation critique, Ottawa, Conseil de recherches en sciences humaines du Canada, avril 1980, 81 p. La gestion dans les années 80: le choix des thèmes pour la recherche en gestion, Rapport de la conférence nationale de la Fédération canadienne des Doyens en gestion et en administration et de l'Association des sciences administratives du Canada, tenue à Toronto, les 29 et 30 décembre 1980, 2 février 1981, 43 p. polycopié. 
Dans un séminaire d'études graduées, par exemple, l'ouvrage permet aux étudiants de passer en revue tout le domaine de l'économie du travail,du droit du travail, de la contribution de la psychologie et de la sociologie aux relations industrielles, et ainsi de suite. Certains chapitres soulignent particulièrement bien l'état de la recherche: ils mentionnent les domaines où les recherches ont été particulièrement fructueuses et ceux qui nécessiteraient un effort plus considérable dans un avenir rapproché.

\section{État de la théorie}

Le troisième aspect, celui de l'état de la théorie, est beaucoup moins développé, pour ne pas dire davantage. Le volume reflète la situation de la discipline elle-même. Plusieurs auteurs l'ont rappelé, nous avons en relations industrielles très peu de théories. Le volume n'apporte rien de neuf à ce sujet: il ne suggère aucune théorie nouvelle. Ceci d'ailleurs n'était pas demandé aux auteurs, on attendait d'eux qu'ils fassent le bilan de ce qui existait au moment où chacun d'eux écrivait son chapitre. Quelques auteurs ont tenu à mentionner les éléments de théorie qui ont pu être développés sur certains sujets particuliers. La tâche de poursuivre dans cette direction revient aux autres ouvrages et aux autres auteurs qui publieront dans l'avenir.

Quelle que soit l'opinion que l'on partage sur la question des relations industrielles comme une théorie ou un champ d'études, il demeure important de développer au moins des théories partielles et peut-être, éventuellement, une théorie générale. Ces théories partielles peuvent provenir des autres disciplines, ou des relations industrielles comme telles.

La plupart des chapitres du livre ont dégagé clairement la contribution de chaque discipline au domaine des relations industrielles et, à l'inverse, la contribution réelle que les relations industrielles ont pu apporter aux disciplines elles-mêmes, par exemple en forçant celles-ci à concentrer leur attention sur d'autres sujets ou sur de nouveaux problèmes.

Il est temps de tourner notre attention du côté des problèmes fondamentaux et des questions de base qui demeurent, concernant la théorie ou le champ des relations industrielles.

\section{PROBLÈMES FONDAMENTAUX}

Quatre questions, déjà mentionnées à propos de l'histoire du volume de l'ACRI, demeurent capitales. Voici un peu de quelle manière elles se présentent à l'heure actuelle.

\section{Définition et objet}

La question de savoir comment on définit les relations industrielles et ce qu'elles doivent contenir demeure toujours pertinente. Les événements 
des deux dernières décennies, dans la plupart des pays industrialisés, ont donné une nouvelle orientation à la question et à la réponse qu'on lui apporte généralement.

Dans la plupart des pays industrialisés, le syndicalisme est en déclin. Le Canada fait exception, où la proportion des travailleurs syndiqués demeure constante, autour de $30 \%$ à $35 \%$ de la main-d'oeuvre salariée. Mais cette stabilité apparente est le résultat de la syndicalisation des employés du secteur public, qui ont dû se syndiquer par suite de lois ou de règlements, et leur syndicalisation a contrebalancé la diminution correspondante du nombre de syndiqués dans le secteur privé.

En plus de ce phénomène sociologique, la plupart des pays industrialisés ont, au cours des années 1970 et 1980 , introduit ou amélioré plusieurs lois sur les normes de travail. Ces lois ont entraîné, pour toute la main-d'oeuvre, syndiquée ou non, une meilleure réglementation des conditions de travail déjà réglementées, et ont accru la réglementation par l'addition de plusieurs objets, comme le licenciement individuel et collectif, la santé et la sécurité au travail, et une multitude d'autres sujets maintenant couverts par les lois.

Ces matières relevaient auparavant exclusivement du domaine de la négociation collective. Aujourd'hui, elles s'appliquent dans tous les lieux de travail, commerciaux et industriels. En vertu de la loi, ces questions préoccupent maintenant toutes les directions du personnel et font partie intégrante de chaque gestion des ressources humaines. Cette situation nouvelle a amené la plupart des écoles et des instituts de relations industrielles à inclure dans leur curriculum l'étude de ces questions qui touchent au personnel et aux ressources humaines. Il est trop tôt pour prédire si l'intégration amènera des modifications éventuelles dans les structures académiques des différentes universités. Mais l'inclusion de ces matières est devenue nécessaire et inévitable.

Un autre phénomène souligne une caractéristique fondamentale de notre domaine d'études, quelle que soit notre opinion personnelle sur la question de savoir si c'est une discipline distincte ou un champ d'études ouvert à de multiples disciplines. Personne ne peut raisonnablement séparer les trois aspects fondamentaux de notre domaine d'études: la réalité ou la pratique, l'étude du secteur tel qu'il se reflète dans les structures académiques, dans les départements et les facultés des universités, et finalement le domaine de la recherche et de la théorie. Une véritable compréhension du développement des relations industrielles doit inclure, directement ou indirectement, la considération de ces trois aspects. Dans cette perspective, j'aimerais mentionner un article particulièrement éclairant, paru récemment dans la revue Relations industrielles, sous le titre suivant: «La théorie des relations industrielles au Canada: une approche historique» ${ }^{6}$. Les trois éléments mentionnés s'y retrouvent: la réalité vécue des relations industrielles, leur enseignement et la théorie qui peut en découler.

6 Gregor MURRAY et Anthony GILES, «La théorie des relations industrielles au Canada: une approche historique», Relations industrielles - Industrial Relations, Québec, PUL, vol. 43, nº 4, 1988, pp. 780-811. 


\section{Discipline ou champ d'études?}

La persistante question à savoir si les relations industrielles sont une discipline propre et de plein droit, ou un champ d'études pour les différentes sciences sociales, demeure présente à l'esprit de bien des universitaires et des praticiens dans le domaine. Quelques-uns considèrent qu'une telle discussion n'est rien d'autre qu'une perte de temps, mais certains croient qu'on peut en tirer des intuitions et une meilleure intelligence du domaine dans son entier.

Il n'est peut-être pas inutile que j'exprime clairement quelle est ma position personnelle sur le sujet, ce que je ne pouvais pas faire dans le livre de l'Association. Comme je les comprends, les relations industrielles sont un champ d'études, fort important d'ailleurs, que je comparerais à la médecine. La médecine a pu être considérée comme une discipline il y a longtemps; elle n'est plus une discipline mais un sujet d'études et de recherche, qui s'est donné comme objectif de découvrir de nouveaux moyens de traiter et de guérir le corps (et l'esprit) humain. La physique, la chimie, l'anatomie, la psychologie et la psychiatrie sont autant de méthodes pour étudier et comprendre le fonctionnement de l'être humain. La médecine, c'est l'ensemble de toute cette recherche pour le mieux-être de l'humanité. Pourquoi ne pourrait-on pas considérer de la même manière les relations industrielles?

Une question importante dans la controverse des relations industrielles comme une discipline ou un champ d'études est la suivante: quel est le rôle propre et la place particulière qui revient à l'approche systémique? Depuis l'ouvrage qui a fait de John Dunlop l'initiateur des préoccupations de cette nature, l'approche systémique ou systématique aux relations industrielles a fait l'objet de multiples études par des universitaires de toutes origines. Pourtant la question fondamentale demeure: le succès connu fait-il de l'approche systémique $l a$ théorie des relations industrielles? Même si cette approche s'est avérée extrêmement fructueuse et un instrument efficace pour aborder les problèmes de base de relations industrielles, je considère que l'approche systémique n'est pas une théorie, mais un instrument précieux pour ordonner et ordonnancer les multiples relations qui peuvent exister entre les nombreux acteurs d'un régime de relations industrielles. En fait, l'approche systémique est née non pas dans les sciences sociales, mais en biologie. Elle est et demeure un moyen puissant de décrire les relations fondamentales qui existent entre les acteurs impliqués et, à ce titre, elle est à l'analyse des corps sociaux en cause et de leur comportement ce que sont les mathématiques par rapport au raisonnement déductif. L'approche systémique est un instrument, elle n'est pas le coeur de nos études.

En fait, la question elle-même soulève quelques interrogations fondamentales: qu'est-ce qu'une science, une théorie, une discipline?

\section{Science, théorie et autres catégories}

Il y a un flot - que dis-je une débâcle sinon une cataracte - de mots qui s'utilisent couramment dans les discussions qui nous préoccupent. Quelques exemples: discipline, méthodologie, théorie, doctrine, lois, systèmes, 
modèles, structures, paradigmes, écoles de pensée, et la liste pourrait continuer. Il semble nécessaire d'introduire un certain ordre entre ces termes avant de s'embarquer dans quelque discussion que ce soit sur les relations industrielles comme une théorie ou un champ d'études. Je suggère les balises suivantes.

Il semble utile de commencer par le petit bout de la lunette. D'abord, il faut reconnaître l'existence de quelques lois; ainsi, en économique, l'observation la plus fondamentale est sans doute la loi de l'offre et de la demande. Pour ne donner qu'un exemple en relations industrielles, la plupart des universitaires et des praticiens reconnaîtraient sans doute l'affirmation suivante comme assez exacte pour en faire une loi, au moins dans les sociétés démocratiques de l'Ouest: "Toute entente volontaire sera plus facilement applicable qu'une décision imposée de l'extérieur.»

Une théorie est un ensemble cohérent de lois vérifiées ou vérifiables permettant de comprendre, d'expliquer et même de prédire divers phénomènes. Une théorie bien connue en économique est celle dite de la productivité marginale. Avant de passer au mot discipline, il y aurait lieu de définir brièvement doctrine, à cause des différences fondamentales qui existent entre théorie et doctrine. Une doctrine se rapporte d'abord et avant tout à certains principes philosophiques, sinon même à une idéologie. Une idéologie est un système d'idées-force ou de croyances qui sert à animer, décrire, expliquer, interpréter ou justifier l'action d'un individu ou d'une collectivité; cette action s'inspire d'un ensemble de valeurs et elle oriente l'activité de la personne ou du groupe qui épouse l'idéologie. Le terme implique une part de coercition venant du groupe, qui a pris sur lui de propager un certain système de pensée et de vie. Une doctrine, bien que généralement moins coercitive, est également orientée vers l'action et le choix de politiques; elle n'est pas intéressée à la seule intelligence et compréhension des événements, ce qui est le propre de la théorie. Un exemple classique en économie c'est la doctrine du laisser-faire. Il peut y avoir des liens entre la théorie et la doctrine, mais fondamentalement, ce sont deux réalités différentes, et elles s'intéressent à la pratique avec des objectifs et des préoccupations opposées.

Une discipline a un sens beaucoup plus étendu et moins précis. Elle désigne une branche de connaissances dans laquelle on se spécialise et qui fait appel à un ensemble particulier de principes et de règles pratiques. Mais voilà qui nous ramène à notre point de départ; nous sommes presque au rouet: qu'est-ce qui fait qu'un ensemble ou une branche de connaissances constitue une unité particulière et se distingue clairement de toute autre? Plusieurs ont tendance, pour répondre à cette question, à se tourner vers les divers départements des différentes universités et à considérer chaque département comme correspondant à une discipline particulière. Mais certaines universités - et la mienne est dans le groupe - ont une autre distinction: elles parlent de département disciplinaire et d'école professionnelle: une école professionnelle, comme la nôtre, peut avoir toutes les caractéristiques et tous les pouvoirs académiques et politiques d'un département; la seule différence réside dans le fait que l'école est rattachée d'une certaine manière à une profession: ses gradués se dirigent normalement vers la pratique de cette profession. En fait, malgré l'identité de pouvoirs, le titre d'école par opposition à département peut, dans certains cas, évoquer une catégorie de rang inférieur. 
Quoi qu'il en soit, la question demeure: qu'est-ce qui distingue une discipline d'une autre? Les définitions courantes se réfèrent à des champs particuliers d'études, mais plusieurs disciplines étudient souvent les mêmes objets, les mêmes comportements, comme c'est le cas en relations industrielles. Je suggère que la méthodologie utilisée serait un meilleur instrument d'identification, même si ce n'est certainement pas le seul. Elle serait un peu, à mon avis, le discriminant par excellence.

Cette question de la méthodologie a beaucoup plus d'implications qu'on ne peut le croire. Elle peut orienter ou réorienter les études et la recherche dans telle ou telle direction. Â l'heure actuelle, en relations industrielles, beaucoup d'études se fondent sur des enquêtes d'attitudes, ce qui correspond à une certaine méthodologie, qui a l'attrait de se traduire facilement en des formules mathématiques. Mais ne se pourrait-il pas que certains chercheurs se laissent prendre par l'instrument et oublient des questions beaucoup plus fondamentales comme celles des causes (le pourquoi des choses), des origines (le point de départ) et des orientations (le point d'arrivée).

Ces dernières questions impliquent des jugements de valeurs. Auparavant, il faut mentionner d'autres mots. On a parlé plus tôt des systèmes et des structures, et de l'approche systémique. On pourrait ajouter d'autres mots, moins bien définis, comme les paradigmes et les écoles de pensée. Relativement souples, ces termes et catégories mentales sont utiles, parce qu'ils permettent d'identifier des regroupements et des tendances dans les principales sources d'explication des phénomènes; ils permettent d'identifier plus facilement sinon d'évaluer les différentes étapes qu'on peut reconnaître dans le développement de la théorie ou des théories en relations industrielles.

\section{Valeurs, objectifs et pratique des R.I.}

Cet aspect des valeurs et des objectifs, que nous avons délibéremment laissé de côté dans le livre de l'ACRI, demeure cependant un des aspects les plus fondamentaux de notre problème. Le volume a été critiqué de diverses manières: on a dit qu'il contenait trop, dans d'autres cas qu'il contenait trop peu, de discussions clairement orientées vers des prises de position et des recommandations de nature politique.

Les relations industrielles ont toujours été un champ d'études fortement tourné vers la pratique. Cela tient à diverses raisons, qu'il n'est pas opportun de développer ici. Mais cela souligne aussi un fait: les professeurs et les chercheurs en relations industrielles peuvent difficilement se détacher complètement de la pratique des relations industrielles.

Je crois que, comme universitaires et chercheurs, il est de notre devoir de concentrer nos efforts pour chercher à comprendre et à expliquer les réalités de relations industrielles, non pas de les orienter dans la pratique. Comme citoyen, nous avons ce droit et peut-être même cette responsabilité. Mais notre premier devoir comme universitaire demeure, à mon avis, du côté de la recherche des explications. 
Je sais très bien que plusieurs considèrent qu'il n'y a pas de position «objective» comme celle qu'impliquent les affirmations précédentes. On dira qu'une telle recherche dite objective comporte elle-même une prise de position et un jugement de valeurs qui favorisent les institutions et le régime en place. Je suis conscient que la ligne est mince et facile à franchir entre ces deux prises de position.

Pourtant, comme étudiant des relations industrielles, je ne peux que souhaiter qu'un grand nombre de chercheurs et de professeurs prennent le risque d'analyser les rapports et les relations qui peuvent exister entre des objectifs sociétaux et des valeurs morales, même sous-entendus, et la plupart des lois et des politiques, sans oublier les analyses «scientifiques» de plusieurs publications universitaires. La tâche est extrêmement difficile et périlleuse. Les universitaires canadiens qui ont publié le volume dont nous discutons n'ont pas voulu s'attaquer à cette dimension majeure et fondamentale. Personnellement, je suis convaincu que cet effort demeure nécessaire pour mieux comprendre nos positions respectives, même pour mieux orienter les politiques publiques en matière de relations industrielles, et surtout pour permettre la construction progressive de théories ou d'une théorie des relations industrielles.

\section{CONCLUSION: LES PROCHAINES ÉTAPES}

Un an après la parution de L'état de la discipline en relations industrielles au Canada, compte tenu des premiers commentaires et réactions, positifs et négatifs, il me semble qu'il n'y a qu'une conclusion possible: il faut poursuivre et aller plus loin. Je suggère que l'effort doit se poursuivre partout en plusieurs directions. Je soumets les trois observations qui suivent comme des exigences minimales pour continuer le travail.

\section{Entente minimale sur le sens des mots}

Il faudrait faire un effort entre nous pour utiliser les mêmes mots dans le même sens. Je ne veux pas dire que nous devons tous être d'accord sur le contenu des définitions, mais qu'il faudrait être consistants avec nousmêmes et le plus possible les uns entre les autres. Une théorie est une théorie et non pas une doctrine; une discipline est une discipline, même si nous ne sommes pas sûrs quels en sont les signes distinctifs. Je souhaite également que l'on s'entende sur une définition large et englobante des relations industrielles, qui inclut la gestion des ressources humaines. Je souhaite également que cette reconnaissance ne soit pas seulement une reconnaissance dans les mots, mais dans les faits également, surtout dans le domaine de la recherche. L'intégration dans les structures universitaires peut exiger plus de temps. 


\section{Questions d'èthique et de valeurs}

Comme nous l'avons rappelé plus haut, il y a une pressante nécessité d'aborder des études sérieuses en matière de valeurs, d'éthique et d'objectifs, tant au niveau de la pratique qu'à celui de la recherche et de l'enseignement. Les conclusions risquent d'être contradictoires. Mais, à long terme, nous pourrons peut-être nous entendre sur quelques objectifs, sur la nécessité d'un cadre de pensée explicite, qu'il s'agisse d'un régime capitaliste, socialiste ou communiste.

\section{Une construction progressive de théories}

Quel que soit notre point de vue personnel sur la controverse entre une discipline et un champ d'études, il me paraît nécessaire d'établir quelques principes fondamentaux ou théories partielles, qui pourraient être généralement acceptés et servir de pierre d'angle pour une éventuelle théorie générale des relations industrielles.

Parce que les relations industrielles sont tellement incarnées dans un contexte local, en particulier le contexte légal et l'environnement économique, une théorie générale ne semble pas possible dans un proche avenir, peut-être jamais. Peut-être pourrait-on envisager de telles théories générales limitées à un pays ou à un système de relations industrielles.

Pour arriver à cette fin, je crois qu'il est essentiel que nous ayons de plus en plus d'études descriptives de la réalité, si nous voulons que nos théories et nos explications soient solidement enracinées dans la pratique des relations industrielles, comme celles-ci évoluent dans nos pays respectifs. Ces monographies seront sans doute réalisées dans une approche de type institutionnaliste, mais elles représentent les études de base qui sont essentielles pour en dégager plus tard quelques lois ou quelques théories partielles.

Dans la même perspective, je suggère qu'il n'y a pas de méthodologie unique qui puisse nous donner une connaissance complète pour expliquer les phénomènes, pour établir leurs origines et leurs orientations propres.

Comme nous l'avons dit plus tôt, je crois que l'approche la plus fructueuse et la plus éclairante consiste à étudier simultanément le développement de la réalité ou de la pratique, le développement des études et de l'enseignement et le développement de la recherche et des théories. Voilà, me semble-t-il, un effort qui convient à des universitaires, mais aussi à des citoyens engagés, puisque une compréhension adéquate du fonctionnement des relations industrielles en pratique est susceptible de conduire à des suggestions de politiques mieux adaptées dans l'avenir. Le projet vaut que nous mettions tous l'épaule à la roue et que nous fassions chacun notre part pour atteindre une meilleure compréhension et connaissance de l'important sujet d'études que nous avons choisi, comme carrière d'étudiant, d'enseignant et de chercheur. 


\section{The State of the Art in Industrial Relations}

\section{A Brief History and a Postscript}

The Canadian Industrial Relations Association (CIRA) has published, during the last academic year, a collection of ten essays entitled The State of the Art in Industrial Relations. The book appeared in the two official languages of Canada, in French and in English.

After a few notes on the history of the project, the author will briefly discuss the major problems involved in its realization and comment on the steps to be taken to move forward in our quest for an industrial relations theory.

\section{CIRCUMSTANTIAL HISTORY}

The book has been in preparation for almost 10 years. The first idea was sparkled by Thomas Kochan's Report on Research Priorities in Industrial Relations, when that report was still in preparation for the United States ${ }^{1}$. Professor Noah Meltz of the University of Toronto discussed with Labour Canada representatives the opportunity of a similar study for Canada. At the annual meeting of CIRA, in 1980, the general assembly gave the executive committee the mandate to pursue the project. A few months later, a Research Policy Committee of CIRA came up with a grandiose design, which involved expenses of close to 200000 Canadian dollars. A few months later, Labour Canada turned down the whole plan.

The project was immediately redirected to a federal granting agency, the Social Sciences and Humanities Research Council of Canada. The Council had shown interest in State of the Art projects. One year later, the granting council offered a meagre $\$ 15000$, and the cheque came in almost another year later, in early 1983 . The money was used for research assistance, typing, photocopy, long distance telephone calls, and very limited travel expenses. The project itself was realized on a strictly voluntary basis by all the contributors, including the steering committee members.

For various reasons, like people away on sabbatical, it took another year to complete an outline for the book and to enlist prominent and willing authors. The project got really on its way in June 1984, at CIRA meeting in Guelph, Ontario. Everybody thought one year or two was a reasonable delay for the book to come out. As is often the case in similar ventures, one or two chapters came in late and publication date had to be postponed. The book was finally launched in August 1988, at the First Industrial Relations Congress of the Americas, in Québec City. The French version of the book was then ready and available for sale; the English version came out a few months later.

This is a very short summary of a long fascinating, but sometimes difficult effort by the Canadian Industrial Relations academic community to come up with a printed presentation of the State of the Art it is practising. The more interesting aspect of the venture lies in the many options the steering committee had to take during these many years of gestation.

1 Thomas A. KOCHAN, Labor-Management Relations Research Priorities for the 1980s, Washington D.C., U.S. Department of Labor, January 1980. 


\section{SUBSTANTIAL HISTORY}

Options had to be made on many aspects. I will briefly discuss three of them of basic importance: the scope of industrial relations, the approach to a State of the Art analysis and the final outline of the project.

\section{The Scope}

The first problem we had to face was the very definition of industrial relations. Even working from an empirical basis, we were facing a major duality. Anglophone universities in Canada tend to have Centres for industrial relations, where the main preoccupation is with collective relationships, i.e. unions, labour law and collective bargaining; the teaching of human resources management is then left to the Schools or Faculties of Management. On the other hand, Francophone universities tend to have distinct and separate Departments of Industrial Relations, where both collective relationship aspects and personnel or human resources management are taught to a definite body of students, registered officially as industrial relations students.

The steering committee agreed that both aspects should be covered in the book, but a statement of that nature in the opening chapter was not enough. Despite suggestions to that effect, every single author tackled his subject according to his own view and emphasized the aspect he was more familiar with in his own teaching and research work. Thus the book has already been accused of being slanted in favour of collective relationships, and of not giving human resources management its due place and rôle.

\section{The Approach}

Even if it was much less encompassing, our second problem was much more difficult to resolve. Everyone agrees that industrial relations is a field for interdisciplinary study and research, but the unanimity does not go much further. We all agreed that the major disciplines should be part of the project, but it was much less clear how they should get into the picture. Very quickly, we were faced with the following choice: to structure the book by issues, and ask every author to use a multidisciplinary approach to discuss the issue under review, or to select a discipline approach and suggest each author to touch on the major issues his own discipline did study or should study.

The steering committee finally concluded the project would be more manageable if we were to take the discipline approach. This leads us into our third problem or difficulty, the actual outline of the book.

\section{The Outline}

Once the decision was made to structure the book by discipline, it was easy to list the disciplines to be definitely included. Economics and law have been the two disciplines more closely related to industrial relations over the whole of the XXth century. More lately, sociology and psychology, and the sub-discipline of organizational behavior, have become major contributors to the understanding of industrial relations; as such they were to be part of the core list. 
But the unanimity stopped there. What about management sciences, history and philosophy, to name but these three? Not everyone agreed that management studies were a recognized discipline; some contended they were in exactly the same position to other disciplines as industrial relations. We finally agreed to include management studies, and history, because of the contribution these two sectors or disciplines had made to the understanding of certain parts of industrial relations. But we left out philosophy, not because the subject was not important: values are everywhere in any problem of industrial relations. Is was rather because we thought it would be impossible to find an author who could present a fairly complete overview of the subject, without alienating too many readers. We felt sensitivity is so high when it comes to goals and values, that we should perhaps avoid that snake pit. After all, this was the project of the whole of a complex and varied Canadian Industrial Relations Association.

On the contrary, we did include a chapter called Mainstream Industrial Relations, because of the important part of the opinion that considers some subjects are not well taken care of by any of the major disciplines. We also felt that the systems approach was so important that it had to be a part of our State of the Art project. We thus asked to write that chapter to a Canadian author who is generally recognized as having pushed further the wellknown and widely-used systems approach developed 40 years ago by John T. Dunlop ${ }^{2}$.

To add a brief footnote to the outline of the book, the committee considered that two of the disciplines had to be subdivided between English Canada and French Canada, because of their specific relationship with culture, namely law and history. In other words, there are two different chapters for each of these disciplines, one concerned with French Canada and the other with English Canada. Together with the introductory and concluding chapters, the total adds up to the 10 chapters the book actually contains.

\section{Discipline or Field?}

That question remained unresolved, and the committee concluded it had to be that way. At an early stage of the project, in 1983, I had volunteered to write an Introduction and Position Paper on the project, which was submitted at CIRA's Annual Meeting in Vancouver. Since I could not attend that meeting, I was told that the argument between those who defended IR as a discipline against IR as a field of study became so intense that it almost jeopardized the whole project.

We could not avoid to mention the problem, because of its basic character. We agreed it had to be presented in the introductory chapter of the book. No specific request was made to any author to discuss the question. It was not that easy to review the matter in the concluding chapter. Despite my own personal opinion, I had to present as forcefully as I could the two sides of the discussion. A project of an association could not antagonize an important part of its membership by taking a definite stand on such a controversial question.

2 John T. DUNLOP, Industrial Relations Systems, New York, Henry Holt, 1958, $339 \mathrm{p}$. 


\section{STATE OF THE ART}

Defining what is a State of the Art study was another problem. When we began our discussions, a certain number of such studies were under way in other fields $\mathbf{s}^{3}$, but there were yet very few publications on the subject ${ }^{4}$. After long discussions we finally concluded that a complete State of the Art analysis should include a state of the knowledge in the field, a state of the theory, and a state of the research. We left it to each author to insist on one aspect or the other, as he wished to do.

\section{State of Knowledge and Research}

The result, as found in the book, is a fair description of the state of the knowledge and of the state of the research on industrial relations according to each discipline considered. These are, I believe, the two strong points of the book.

I have used the book in a graduate seminar, and with the help of its various chapters the students were able to do a through review of labour economics, of labour law, of the contribution of sociology and psychology to industrial relations, and so on. Certain chapters also underline extremely well the state of the research, mentioning the areas where further research is more immediately needed.

\section{State of the Theory}

The third aspect, the state of the theory, is much less developed, if it is at all. The book reflects the very situation of our field. As is mentioned by a few of the authors, we have little by way of theory. In a real sense, the contribution of the book to theory-building in industrial relations is nil: nothing new of that nature is being created. This was not asked to the authors, but simply an account-taking of what existed when each one wrote his own chapter. Some of the authors do mention the bits of theory that have been developed on a few specific topics. The task of moving further in that direction has been left for other books and authors.

Whatever one's opinion on the question of industrial relations as a theory or a field of study, developing theories and eventually a general theory remains an important task to tackle. These bits of theory may come from a number of disciplines, or may be specific to industrial relations.

3 Two were published later under the following titles: Law and Learning, Report to the Social Sciences and Humanities Research Council of Canada by the Consultative Group on Research and Education in Law, Ottawa, Social Sciences and Humanities Research Council of Canada, 1988, x-186 p.; The development of Political Science in Canada: Fifty Years in the History of a Discipline, edited by Michael STEIN, John TRENT and André DONNEUR.

4 Consultative Group on Research and Graduate Education in Business Management and Administrative Studies, University Management Education and Research: $A$ Developing Crisis, Ottawa, Social Sciences and Humanities Research Council of Canada, April 1980, 81 p. Managing in the 1980s: Choosing Themes for Management Research, Report of the National Conference of the Canadian Federation of Deans of Management and Administrative Studies and the Administrative Sciences Association of Canada, held in Toronto, December 29-30, 1980, February 2, 1981, 38 p. Multicopied. 
Most chapters of the book have specified what contribution each discipline has made to industrial relations and, vice versa, what contribution industrial relations have make to the discipline itself, forcing it to focus its attention on new or different subjects.

We should now turn our attention to the future, and look at the most fundamental or basic questions that remain to be considered, concerning the theory or the field of industrial relations.

\section{BASIC QUESTIONS}

Four questions already encountered in discussing the story of CIRA's book remain prominent. We will try to put them in their current perspective.

\section{Definition or Scope}

The question of what comes under industrial relations is still relevant. Events of the last two decades have definitely oriented the direction of the answer.

Unionization is on the decline in most industrialized countries. One exception is Canada, where the proportion of unionized workers remain around $30 \%$ or $35 \%$ of the total workforce. But this stability has come by law or some other kind of ruling, forcing public sector employees to join a union, which has counterbalanced a net decline in private sector unionization.

Together with this sociological fact, most industrialized countries have begun, in the $70 \mathrm{~s}$ and $80 \mathrm{~s}$, to implement or to better their work standards legislation. This has meant, for the whole work force, unionized or not, better regulation of their working conditions already regulated, and new regulations on more and more topics, like individual and collective dismissal, health and safety, and a variety of other subjects now covered by law.

All these subjects were previously the exclusive domain of collective bargaining. Now they apply in all commercial and industrial premises; thus by law they are part and parcel of human resources management. This new situation makes it imperative for industrial relations to include in its curriculum the study of these personnel and human resources questions. It is too early to predict how the integration will be translated into the academic structures of our various universities. But their inclusion somewhere has become a necessity.

The same phenomenon underlines a basic characteristic of our field of study, whatever our personal opinion on the question of a specific discipline or a multidisciplinary approach to the field. No one can reasonably separate the three basic aspects of our field of study: the reality or practice, the study of the field as incarnated in our academic structures, in university departments and faculties, and finally the domain of research and theory. A thourough understanding of the development of industrial relations must refer, directly or indirectly, to these three aspects. In that perspective, I would like to mention a particularly enlightening article, which was 
published recently in Relations industrielles under the expressive title of «Towards an Historical Understanding of Industrial Relations Theory in Canada» ${ }^{5}$. The three elements are there: the reality, the teaching and the possible theory.

\section{A Discipline or a Field of Study?}

The ever-haunting question whether industrial relations is a discipline in its own right or a field of study to be investigated by a variety of social sciences is still very much present in the minds of a good number of people. Some consider it is a waste of time, but others are of the opinion that some insights and a better intelligence of the whole domain may be obtained from such a discussion.

Perhaps I should say here what is my own personal position, which I could not express in CIRA's book. In my understanding, industrial relations is a field of study, and a very meritorious one, which I would compare with medicine. Medicine may have been considered a discipline, a long time ago. It is no more one discipline, but a subject for study and research, with a view to discover new ways to cure and treat the human body. Physics, chemistry, anatomy, psychology and psychiatry are all different ways of studying and better understanding the functioning of the human body. Medicine is any part of the search for the better-being of humanity. The field of industrial relations can be looked at much in the same way.

One important question remains to be considered in the controversy of IR as a discipline or as a field: it is the rôle and place of the systems approach. Ever since the publication of John Dunlop's seed-work in 1958, the systems or systematic approach to industrial relations has been studied and used by many scholars in the field, and very fruitfully at that. Still the basic question remains: does this success make the systems approach the theory of industrial relations? Even if this approach has been the most fruitful and successful way of tackling basic IR problems, I submit that the systems approach is not a theory, but a useful device to bring some order in the various relationships that may exist between the numerous actors involved. In fact, it first appeared not in the social sciences but in the biological studies. It is and remains a very powerful means to describe the basic relationships between the various actors of the drama, and, in that sense, it is to the analysis of social bodies and their behaviour like mathematics to deductive reasoning. I look at it as an instrument, not as the meat itself of our studies.

In fact, the question raises a more fundamental one: what is a science, a theory, a discipline?

\section{Science, Theory et al.}

There is a stream, better a flood if not a cataract, of words that are used in our discussions. Just to give a few examples: discipline, methodology, theory, doctrine, laws, systems, models, structures, paradigms, schools of thought, and the list could continue. It would seem necessary to put these terms in some order before engaging into any discussion of IR as a theory or as a field. I suggest the following guidelines.

5 Gregor MURRAY and Anthony GILES, "Towards an Historical Understanding of Industrial Relations Theory and Canada», Relations industrielles - Industrial Relations, Québec, PUL, Vol. 43, No. 4, 1988, pp. 780-811. 
It might help to begin with the smaller end of the telescope. First, some basic laws may be recognized. Thus, in economics, the most fundamental observation is probably the law of supply and demand. To give but one possible example in industrial relations: many I.R. scholars and practitioners might recognize the following statement as significant enough to accept it as a law, at least in Western democratic societies: «Any voluntary agreement will be more readily applicable than an imposed settlement.»

A theory is a coherent set or collection of verified or verifiable laws, which serve to understand, explain and predict phenomena in a certain field. A well-known example in economics is the marginal-productivity theory. Before moving to a discipline, the work doctrine must be considered, because of the differences it has with theory. A doctrine relates first and foremost to certain philosophical principles, if not ideologies. An ideology is a system or series of basic beliefs, which operate as a source of power and forceful conviction to act in a certain way, and pursue certain specific goals. The work ideology suggests a part of coercion from a group, which has taken unto itself to propagate a definite scheme of thinking and action. A doctrine, although generally less coercive, is still policy-oriented, and not interested only in understanding and explaining, which is the proper of a theory. The classical example in economics is the doctrine of laissez-faire. There may be links between theory and doctrine, but basically the two are different realities, and they approach practice with opposite goals and preoccupations.

Discipline seems to have a much looser meaning, the word being used for a package or bundle of knowledge in a definite field. Then we are back to our first question: what makes a certain package of knowledge distinctly identifiable from any other? The tendency might be to look at university departments, and to consider each department as corresponding to a specific discipline. But some universities and my own is in that category - have another distinction: they speak of disciplinary Departments and of professional Schools: a professional School may have all the academic and political powers of a Department; the difference is that a School is directly related to a «profession», its graduates going normally to the practice of that profession; that very fact puts a School, as opposed to a Department, in a special if not in an inferior category.

Be that as it may, the question remains: what makes and identifies a discipline? The most common definitions point to specific fields of studies, but many disciplines study the same fields, as it has been the case regarding industrial relations. I suggest that the methodology used is a better identifying instrument, although certainly not the only one. I am inclined to look at methodology as the discriminant of a discipline.

This question of methodology is much more pervasive than it might seem. It may orient or reorient studies and research in a certain field. Currently, in industrial relations, many studies are based on attitudinal surveys, which is a kind of methodology, with the attractive characteristic of being easily translated into mathematical formulas. I suggest that some researchers might be obsessed by the instrument, and might forget the more basic and fundamental questions of why, where from and where to.

Before considering expressly these more basic questions, a few other words must be thrown in. Systems and structures have been discussed earlier. We could add a loosely-defined group of words, like paradigms and schools of thought. Highly 
flexible in meaning, these categories are useful, because they allow for certain regroupings or tendencies of major explanatory devices; they make it easier to identify if not evaluate the various steps in theory-building in industrial relations.

\section{Values, Goals and Practice}

This aspect, which was left out of CIRA's book, remains probably the most fundamental one. Criticism has been directed at the book, saying both that there is too much or too little of it, especially with regards to policy-oriented discussions.

Industrial relations has always been considered to be and has always been a practice-oriented field a study. It has been so for a whole set of reasons, which it is not necessary to develop here; but it underlines a fact: industrial relations professors and researchers can hardly set themselves completely apart from the practice of industrial relations.

I submit that as academics and university professors, it is our duty to try to concentrate our efforts on understanding and explaining industrial relations realities, not on orienting them in practice. As citizens, we have that right and perhaps that responsibility. But our duty as academics lies in the field of explaining.

I very well know that a good many people consider this «objective» position as impossible to achieve; they argue that it is in itself a value-laden position and a kind of ideology. I realize the line between the two positions is very thin, and that one can easily move from one side to another.

Still, as a student of industrial relations, I would hope that a good number of industrial relations researchers and professors would take the risk of analyzing the relationship that may exist between certain societal goals and values, sometimes unspecified, and many laws and policies, as well as in many otherwise scholarly publications. This is a difficult job. The Canadian scholars, who have undetaken to publish that book on the State of the Art in their country, have withdrawn from that major and fundamental task. Personally I am convinced that it is still necessary for a better understanding of our positions, and eventually to permit the possibility of building some reasonable theory and/or theories of industrial relations.

\section{CONCLUSION OR STEPS TO BE TAKEN}

One year after completing the Canadian State of the Art project, considering the first comments and reactions, both positive and negative, there is only, I feel, one possible conclusion. The work must be carried out further. I suggest that there are many efforts to be continued. I present the three following observations as minimal requirements to that end or purpose.

\section{Minimal Agreement on Words}

An effort should be made between ourselves to use the same words in the same meaning. I do not mean to say that we should all agree on the content of the definitions, but that we should be consistent with one another. A theory is a theory, and not a doctrine, and a discipline is a discipline, whether we are sure or not of what are its distinctive requirements. 
I also hope there will be some agreement on a rather extensive definition of industrial relations, including human resources management. Hopefully this recognition will not be only in words, but in facts also, especially in the domain of research. The integration at the teaching level in our universities may be longer to come.

\section{Ethical Approaches}

As mentioned earlier, there is a pressing necessity for serious studies in goals and values, both at the level of practice, and that of research and teaching. Conclusions may be conflicting, but in the long run, we may agree at least on a few goals, in a clearly-expressed framework of thought, be it a capitalist, welfare, socialist or communist State.

\section{A Progressive Build-up of Theories}

Whatever one's position on the controversy between a discipline and a field, it would seem necessary to try and formulate some fundamental principles or limited theories, which could be fairly commonly accepted, and serve as building stones for an eventual general theory of industrial relations.

Because industrial relations are so dependent on the local context, especially on the legal framework and the economic environment, a general theory is not likely to be possible in the near future. Perhaps some tentative constructions of that nature could be made within each country, or within each industrial relations system.

In order to realize such a goal, I personally feel that accurate descriptive studies are still and will always be needed, if we wish our theories and understanding to be rooted solidly in the industrial relations practice, as it evolves in our respective countries. These monographs, worked out in an institutionalist approach, would represent the solid ground-work on which a few laws or small bits of theory could be built.

In the same perspective, I suggest that no single methodology can give us a full and complete knowledge of the whys, the where-froms and the where-tos in industrial relations.

Again, as mentioned earlier, I suggest that perhaps the most fruitful and enlightening approach would be to look simultaneously at the development of reality or practice, at the development of study and teaching, and at the development of theories and research. This is, I believe, a fitting and suitable effort for us as academics, but also as citizens, since a proper understanding of the functionning of industrial relations in practice is likely to be the most fruitful source of policy suggestions for the future. It is a worthwhile effort for all of us to set one's shoulder to the wheel, and do our small part for a better understanding of the important subject of study we have chosen, as a career of learning, teaching and research. 